Pacific Journal of Mathematics

IDEALIZER AND NONSINGULAR RINGS 


\title{
IDEALIZERS AND NONSINGULAR RINGS
}

\author{
K. R. GOODEARL
}

This paper deals with the relationship between a ring $T$ and the idealizer $R$ of a right ideal $M$ of $T$. [The ring $R$ is the largest subring of $T$ which contains $M$ as a two-sided ideal.] Assuming $M$ to be a finite intersection of maximal right ideals of $T$, the properties of $T$ and $R$ are shown to be very similar. The main theorem of the first section shows that under these hypotheses the right global dimensions of $T$ and $R$ almost always coincide. In the second section, where $T$ is assumed to be a nonsingular ring, the major theorem asserts that the singular submodule of every $R$ module is a direct summand if and only if the corresponding property holds for $T$-modules.

We assume throughout the paper that all rings are associative with identity, and that all modules are unitary. Unless otherwise noted, all modules are right modules.

1. Idealizers. This section is concerned with idealizers in arbitrary rings, and is based on the work of J. C. Robson in [7].

Given a ring $T$ and a right ideal $M$ of $T$, the idealizer of $M$ in $T$ is the set $R=\{t \in T \mid t M \leqq M\}$, which is easily seen to be the largest subring of $T$ which contains $M$ as a two-sided ideal. The aim of this investigation is to discover properties of $T$ which carry over to $R$ (and vice versa).

We shall mainly consider the case when $M$ is a finite intersection of maximal right ideals of $T$; following [7], we say in this case that $M$ is a semimaximal right ideal of $T$. Equivalently, $M$ is a semimaximal right ideal of $T$ if $T / M$ is a semisimple right $T$-module, i.e., a module which is a sum of simple submodules. In accordance with this terminology, we use the term "semisimple ring" to refer to a ring which is semisimple as a module over itself, rather than a ring whose Jacobson radical is zero.

The concept of the idealizer of $M$ is of course not needed if $M$ is already a two-sided ideal of $T$, i.e., if $T M=M$. When $M$ is maximal, the only other possibility is $T M=T$, and in general this condition seems to be required for some proofs. Fortunately, [7, Proposition 1.7] allows us to assume it without loss of generality: Assuming that $M$ is a semimaximal right ideal of $T$, then there is another semimaximal right ideal $M^{\prime}$, containing $M$, such that $T M^{\prime}=T$ and the idealizers of $M$ and $M^{\prime}$ coincide. 
Thus we assume throughout this section that $M$ is a semimaximal right ideal of $T$ satisfying $T M=T$.

Proposition 1. [Robson] (a) $R / M$ is a semisimple ring.

(b) $T / R$ is a semisimple right $R$-module.

(c) $T$ is a finitely generated projective right $R$-module.

(d) The natural map $T \otimes_{R} T \rightarrow T$ is an isomorphism.

Proof. (b), (c), and (d) are contained in Corollary 1.5 and Lemma 2.1 of [7], while (a) follows from the observation [7, Proposition 1.1] that $R / M$ is isomorphic to the endomorphism ring of the right $T$-module $T / M$.

A simple consequence of (d) is that for any modules $A_{T}$ and ${ }_{T} B$, the natural map $A \bigotimes_{R} B \rightarrow A \bigotimes_{T} B$ is an isomorphism, from which we infer that the following maps are also isomorphisms: $A \otimes_{R} T \rightarrow A$, $T \bigotimes_{R} B \rightarrow B, A \rightarrow A \bigotimes_{R} T, B \rightarrow T \bigotimes_{R} B$. Then for any modules $A_{T}$ and $C_{T}$ we conclude using the isomorphisms $A \rightarrow A \otimes_{R} T$ and $C \rightarrow$ $C \otimes_{R} T$ that $\operatorname{Hom}_{R}(A, C)=\operatorname{Hom}_{T}(A, C)$. Given these observations and the projectivity of $T_{R}$, a straightforward induction establishes the following results:

Proposition 2. (a ) $\operatorname{Tor}_{n}^{R}(A, B) \cong \operatorname{Tor}_{n}^{T}(A, B)$ for all $A_{T},{ }_{T} B$ and all $n>0$.

(b) $\operatorname{Ext}_{R}^{n}(A, C) \cong \operatorname{Ext}_{T}^{n}(A, C)$ for all $A_{T}, C_{T}$ and all $n>0$.

These results suggest comparing the global dimensions of $R$ and $T$, which is done in [7, Theorem 2.9] for the case when $T$ is right noetherian: Provided that $R \neq T$, then

$$
\text { r. gl. } \operatorname{dim} .(R)=\max \{1, \text { r.gl. } \operatorname{dim} .(T)\} \text {. }
$$

In Theorem 5 we shall remove the noetherian restriction on this theorem, but first two intermediate results are needed.

The key to the next two propositions is a consideration of the module $J T / J$, where $J$ is a right ideal of $R$. There is an epimorphism $f: F \rightarrow J T / J$ for some direct sum $F$ of copies of $T / R$, and we see from Proposition 1 that $F$ is a semisimple right $R$-module, hence ker $f$ must be a summand of $F$. Thus $J T / J$ is isomorphic to a summand of a direct sum of copies of $T / R$. For the proof of Theorem 10, we must notice that this same conclusion follows when $J$ is an $R$-submodule of a right $T$-module.

Proposition 3. $T$ is a flat left $R$-module. 
Proof. The natural maps $R \bigotimes_{R} T \rightarrow T \bigotimes_{R} T \rightarrow T$ and $T \bigotimes_{R} T \rightarrow T$ are both isomorphisms; hence $R \bigotimes_{R} T \rightarrow T \bigotimes_{R} T$ is an isomorphism. Inasmuch as $T_{R}$ is projective, it follows that $\operatorname{Tor}_{1}^{R}(T / R, T)=0$. Now given any right ideal $J$ of $R, J T / J$ is isomorphic to a summand of a direct sum of copies of $T / R$, from which we infer that $\operatorname{Tor}_{1}^{R}(J T / J, T)=0$. According to Proposition 2 we also have $\operatorname{Tor}_{1}^{R}(T / J T, T)=0$, whence $\operatorname{Tor}_{1}^{R}(T / J, T)=0$. Thus $J \bigotimes_{R} T \rightarrow T \bigotimes_{R} T$ is injective, hence $J \bigotimes_{R} T \rightarrow$ $R \otimes_{R} T$ must be injective.

We shall use the notation $p d_{R}(A)$ to stand for the projective dimension of an $R$-module $A$.

Proposition 4. If $J$ is any right ideal of $R$, then $\operatorname{pd}_{R}(J)=$ $p d_{T}(J T)$.

Proof. Since ${ }_{R} T$ is flat, the tensor product of $T$ with any projective resolution of $J_{R}$ yields a projective resolution of $\left(J \otimes_{R} T\right)_{T}$; thus $p d_{T}\left(J \otimes_{R} T\right) \leqq p d_{R}(J)$. The flatness of ${ }_{R} T$ also implies that $J \bigotimes_{R} T \cong J T$; hence we get $p d_{T}(J T) \leqq p d_{R}(J)$.

In view of the projectivity of $T_{R}$ and $R_{R}, p d_{R}(T / R) \leqq 1$. Inasmuch as $J T / J$ is isomorphic to a summand of a direct sum of copies of $T / R$, we obtain $p d_{R}(J T / J) \leqq 1$. Examining the long exact sequence of Ext, we infer from this that $p d_{R}(J) \leqq p d_{R}(J T)$. Recalling again that $T_{R}$ is projective, we see that any projective resolution of $(J T)_{T}$ is also a projective resolution of $(J T)_{R}$, from which we conclude that $p d_{R}(J T) \leqq p d_{T}(J T)$. Thus $p d_{R}(J) \leqq p d_{T}(J T)$.

[After the preparation of this paper, Professor Robson informed the author that he too had obtained the following theorem, which appears in [8, Theorem 2.8].]

Theorem 5. If $R \neq T$, then r.gl. $\operatorname{dim} .(R)=\max \{1, \mathrm{r} . \mathrm{gl} . \operatorname{dim} .(T)\}$.

Proof. If r.gl. $\operatorname{dim} .(R)>0$, then from Proposition 4 we obtain r.gl. $\operatorname{dim} .(R)=1+\sup \left\{p d_{R}(J) \mid J \leqq R_{R}\right\} \leqq 1+\sup \left\{p d_{T}(K) \mid K \leqq T_{T}\right\}=$ $\max \{1, \mathrm{r} . \mathrm{gl}$. dim. $(T)\}$. On the other hand, it is immediate from Proposition 2 that $\mathrm{r}$.gl. $\operatorname{dim} .(T) \leqq \mathrm{r}$. gl. $\operatorname{dim} .(R)$. Thus it only remains to prove that r. gl. $\operatorname{dim} .(R) \geqq 1$.

In view of the assumption $R \neq T$, we see that $M$ cannot be a two-sided ideal of $T$; hence $1 \notin M$ and $M<R$. Inasmuch as $T M=T$, it follows that the map $R \bigotimes_{R}(R / M) \rightarrow T \bigotimes_{R}(R / M)$ is not injective, from which we conclude that ${ }_{R}(R / M)$ is not flat. Thus $\operatorname{GWD}(R)>0$; hence r. gl. $\operatorname{dim} .(R)>0$.

For weak dimension, the proofs of Proposition 4 and Theorem 5 
can be used, mutatis mutandis, to prove the following theorem:

Theorem 6. If $R \neq T$, then $\operatorname{GWD}(R)=\max \{1, \operatorname{GWD}(T)\}$.

2. Nonsingular rings. In this section we shall assume that $T$ is a nonsingular ring and then investigate the relationship between singular and nonsingular modules over $T$ and $R$. First we recall the relevant definitions: Letting $\mathscr{S}(T)$ denote the collection of essential right ideals of $T$, then the singular submodule of a right $T$-module $A$ is the set $Z_{T}(A)=\{x \in A \mid x I=0$ for some $I \in \mathscr{S}(T)\}$. We say that $A$ is singular [nonsingular] provided $Z_{T}(A)=A\left[Z_{T}(A)=0\right]$. The singular submodule of $T_{T}$ is a two-sided ideal of $T$, called the right singular ideal of $T$ and denoted $Z_{r}(T) ; T$ is a right nonsingular ring if $Z_{r}(T)=0$. Analogous definitions and notations hold for $R$ and its modules.

Throughout this section, we assume that $T$ is a right nonsingular ring and that $M$ is an essential right ideal of $T$, and we investigate the idealizer $R$ of $M$. For all but the next two propositions, we make the additional assumptions that $M$ is a semimaximal right ideal of $T$ and that $T M=T$.

Proposition 7. (a) $\mathscr{S}(T)=\left\{K \leqq T_{T} \mid K \cap R \in \mathscr{S}(R)\right\}$.

(b) $\mathscr{S}(R)=\left\{J \leqq R_{R} \mid J M \in \mathscr{S}(T)\right\}$.

(c) $Z_{T}(A)=Z_{R}(A)$ for all $A_{T}$.

(d) $Z_{r}(R)=Z_{R}(T)=0$.

Proof. (a) Suppose that $K \in \mathscr{S}(T)$ and $A \leqq R_{R}$ such that $A \cap(K \cap R)=0$. Then $A M \cap K=0$, whence $A M=0$ [because $A M$ is a right ideal of $T$ and $K \in \mathscr{S}(T)]$. Thus $A \leqq Z_{r}(T)=0$ and so $K \cap R \in \mathscr{S}(R)$.

Now let $K \leqq T_{T}$ and assume that $K \cap R \in \mathscr{S}(R)$. If $A \leqq T_{T}$ and $A \cap K=0$, then from $(A \cap R) \cap(K \cap R)=0$ we obtain $A \cap R=0$, hence $A \cap M=0$. Thus $A=0$ and so $K \in \mathscr{S}(T)$.

(b) If $J \leqq R_{R}$ and $J M \in \mathscr{S}(T)$, then $J M \in \mathscr{S}(R)$ by (a), whence $J \in \mathscr{S}(R)$.

Now consider any $J \in \mathscr{S}(R)$. Inasmuch as $M \in \mathscr{S}(T)$ and $Z_{r}(T)=0$, the left annihilator of $M$ in $T$ is zero. In particular, it follows that every nonzero element of $J$ has a nonzero right multiple in $J M$. Thus $J M$ is an essential $R$-submodule of $J$, hence $J M \in \mathscr{S}(R)$, and then $J M \in \mathscr{S}(T)$ by (a).

(c) follows directly from (a) and (b).

(d) According to (c), $Z_{R}(T)=Z_{r}(T)=0$, and then $Z_{r}(R)=0$ also. 
Let $Q$ denote the maximal right quotient ring of $T$. From [3, Theorem $1+2$, p. 69] we obtain the following information: $Q_{T}$ is an injective hull for $T_{T}, Q$ is a von Neumann regular ring, and $Q_{Q}$ is injective. Note that $T \cap Z_{T}(Q)=Z_{r}(T)=0$, from which we obtain $Z_{T}(Q)=0$.

Proposition 8. $Q$ is also the maximal right quotient ring of $R$.

Proof. We first show that $Q$ is a right quotient ring of $R$, i.e., that $Q_{R}$ is a rational extension of $R_{R}$. (See [3, pp. 58, 64] for the definitions.) Inasmuch as $Z_{r}(R)=0$, [3, Proposition 5, p. 59] says that it suffices to prove that $Q_{R}$ is an essential extension of $R_{R_{0}}$. Thus consider any $A \leqq Q_{R}$ such that $A \cap R=0$. Then $A M \cap M=0$ 。 Since $M$ is an essential right ideal of $T$, it must be an essential $T$-submodule of $Q$, so that we obtain $A M=0$ and $A \leqq Z_{T}(Q)=0$. Therefore, $Q$ is a right quotient ring of $R$; hence we may assume that $Q$ is a subring of the maximal right quotient $\operatorname{ring} P$ of $R$. The injectivity of $Q_{Q}$ implies that $P_{Q}=Q \oplus B$ for some $B$. Then from $R \cap B=0$ we infer that $B=0$ and $P=Q$.

In view of Proposition 8, we may refer to [3, Theorem $1+2$, p. 69] again and conclude that $Q_{R}$ is an injective hull for $R_{R}$. Now we obtain from [5, Proposition 1, p. 427] the following alternate description of the singular submodule of a right $R$-module $A: Z_{R}(A)=$ $\bigcap\left\{\operatorname{ker} f \mid f \in \operatorname{Hom}_{R}(A, Q)\right\}$. In particular, $A$ is singular if and only if $\operatorname{Hom}_{R}(A, Q)=0$, from which we conclude that any extension of a singular module by a singular module is singular.

N.B.-From this point on, the assumption that $M$ is a semimaximal right ideal of $T$ satisfying $T M=T$ will hold.

It follows from Proposition 7 that every nonsingular right $T$ module is also a nonsingular right $R$-module. A partial converse is provided in the next proposition: Any nonsingular right $R$-module can be canonically embedded in a nonsingular right $T$-module.

Proposition 9. If $A_{R}$ is nonsingular, then the natural map $A \rightarrow A \bigotimes_{R} T$ is injective and $\left(A \bigotimes_{R} T\right)_{T}$ is nonsingular.

Proof. In view of the discussion following Proposition 8, the intersection of the kernels of the homomorphisms from $A$ into $Q_{R}$ must be zero. Thus we may assume that $A$ is a submodule of some direct product $B$ of copies of $Q$.

Since $Q$ is a nonsingular right $T$-module, so is $B$. We now get a natural map $A \bigotimes_{R} T \rightarrow B \bigotimes_{R} T \rightarrow B$, and the composition $A \rightarrow A \bigotimes_{R} T \rightarrow B$ 
is just the inclusion map, whence $A \rightarrow A \otimes_{R} T$ must be injective. Also, we see from the flatness of ${ }_{R} T$ that $A \bigotimes_{R} T \rightarrow B \bigotimes_{R} T$ is injective. Since $B \bigotimes_{R} T \rightarrow B$ is an isomorphism, we infer that $A \otimes_{R} T \cong A T$; hence $\left(A \bigotimes_{R} T\right)_{T}$ is nonsingular.

We say that $R$ is a splitting ring provided that for any right $R$-module $A, Z_{R}(A)$ is a direct summand of $A$. It is noted in [1, Proposition 1.12] that $R$ is a splitting ring if and only if $\operatorname{Ext}_{R}^{1}(A, C)=0$ for all nonsingular $A_{R}$ and all singular $C_{R}$.

THEOREM 10. $R$ is a splitting ring if and only if $T$ is a splitting ring.

Proof. Suppose that $R$ is a splitting ring. Given a nonsingular right $T$-module $A$ and a singular right $T$-module $C$, it follows from Proposition 7 that $A_{R}$ is nonsingular and $C_{R}$ is singular. Thus $\operatorname{Ext}_{R}^{1}(A, C)=0$; hence from Proposition 2 we obtain $\operatorname{Ext}_{T}^{1}(A, C)=0$.

Now assume that $T$ is a splitting ring. Given a nonsingular module $A_{R}$ and a singular module $C_{R}$, we must show that $\operatorname{Ext}_{R}^{1}(A, C)=$ 0 . It suffices to prove that $\operatorname{Ext}_{R}^{1}(A, C / C M)=0$ and $\operatorname{Ext}_{R}^{1}(A, C M)=0$. Inasmuch as $M^{2}=M T M=M T=M$, we may thus assume without loss of generality that either $C M=0$ or $C M=C$.

Case I. $C M=0$. We first show that $\operatorname{Tor}_{1}^{R}(A, R / M)=0$.

According to Proposition 9, we may assume that $A$ is an $R$-submodule of a nonsingular right $T$-module $B$. The natural map $T \otimes_{R} M \rightarrow$ $T \otimes_{R} R \rightarrow T$ is injective because $T_{R}$ is projective; hence in view of the condition $T M=T$ we see that $T \bigotimes_{R} M \rightarrow T$ is an isomorphism. Thus $A T \bigotimes_{T} T \bigotimes_{R} M \rightarrow A T \bigotimes_{T} T$ is an isomorphism; equivalently, $A T \otimes_{R} M \rightarrow A T$ is an isomorphism.

Inasmuch as the natural map $R \bigotimes_{R} M \rightarrow T \bigotimes_{R} M \rightarrow T$ is injective, $R \otimes_{R} M \rightarrow T \otimes_{R} M$ must be injective. In light of the projectivity of $T_{R}$, we obtain from this that $\operatorname{Tor}_{1}^{R}(T / R, M)=0$. Now since $A T / A$ is isomorphic to a summand of a direct sum of copies of $T / R$, we must have $\operatorname{Tor}_{1}^{R}(A T / A, M)=0$. Therefore, the map $A \bigotimes_{R} M \rightarrow A T \otimes_{R} M \rightarrow A T$ is injective, hence $A \bigotimes_{R} M \rightarrow A \bigotimes_{R} R$ is injective. $\operatorname{Thus~}^{\operatorname{Tor}_{1}^{R}}(A, R / M)=0$.

Now consider any short exact sequence $E: 0 \rightarrow C \rightarrow B \rightarrow A \rightarrow 0$. Since $\operatorname{Tor}_{1}^{R}(A, R / M)=0$, we obtain another exact sequence $E^{*}: 0 \rightarrow$ $C \rightarrow B / B M \rightarrow A / A M \rightarrow 0$. The sequence $E^{*}$ splits because $R / M$ is a semisimple ring, hence $E$ splits.

Case II. $C M=C$. Here $C \cong P / J$ for some direct sum $P$ of copies of $M$ and some $R$-submodule $J$ of $P$. To prove that $\operatorname{Ext}_{R}^{1}(A, C)=0$, it suffices to show that $\operatorname{Ext}_{R}^{1}(A, P / J M)=0$ and $\operatorname{Ext}_{R}^{2}(A, J / J M)=0$.

Inasmuch as $M \in \mathscr{S}(R), J / J M$ is a singular right $R$-module. Choos- 
ing an exact sequence $0 \rightarrow K \rightarrow F \rightarrow A \rightarrow 0$ with $F_{R}$ free, we have $\operatorname{Ext}_{R}^{2}(A, J / J M) \cong \operatorname{Ext}_{R}^{1}(K, J / J M)$. Since $Z_{r}(R)=0, F$ and thus $K$ are nonsingular; hence $\operatorname{Ext}_{R}^{1}(K, J / J M)=0$ by Case $I$. Therefore, $\operatorname{Ext}_{R}^{2}(A, J / J M)=0$.

All that remains is to show that $\operatorname{Ext}_{R}^{1}(A, D)=0$, where $D=$ $P / J M$. Inasmuch as $P$ is a right $T$-module and $J M$ is a $T$-submodule of $P, D$ is a right $T$-module. Since $P / J$ and $J / J M$ are both singular $R$-modules, it follows from the discussion after Proposition 8 that $D_{R}$ must be singular. Thus from Propositions 7 and 9 we obtain that $D_{T}$ is singular and $\left(A \otimes_{R} T\right)_{T}$ is nonsingular.

Given any exact sequence $0 \rightarrow D \rightarrow B \rightarrow A \rightarrow 0$, we get a commutative diagram with exact rows as follows:

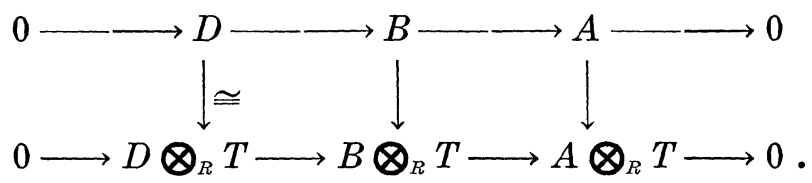

The bottom row splits because $T$ is a splitting ring; hence the top row splits. Therefore, $\operatorname{Ext}_{R}^{1}(A, D)=0$.

One special case of Theorem 10 has been proved in [4]. The authors start with a left and right principal ideal domain $C$ such that $C$ is a simple ring but not a division ring, and such that every simple right $C$-module is injective. (Examples of such rings are constructed in [2].) Then they choose a maximal right ideal $M$ of $C$ and prove that the idealizer $I$ of $M$ in $C$ is a splitting ring [Lemma 2].

It is not hard to prove that every singular right $C$-module is semisimple, and hence that every singular right $C$-module is injective. (Details may be found in [6, Chapter 3].) Thus $C$ is certainly a splitting ring. The right ideal $M$ is nonzero because $C$ is not a division ring; hence from the simplicity of $C$ we obtain $C M=C$. Also, $C$ is a right Ore domain, from which it follows easily that $M$ is an essential right ideal of $C$. Thus it now also follows from Theorem 10 that $I$ is a splitting ring.

\section{REFERENCES}

1. V. C. Cateforis and F. L. Sandomierski, The singular submodule splits off, J. Algebra, 10 (1968), 149-165.

2. J. H. Cozzens, Homological properties of the ring of differential polynomials, Bull. Amer. Math. Soc., 76 (1970), 75-79.

3. C. Faith, Lectures on Injective Modules and Quotient Rings, Springer-Verlag Lecture Notes No. 49, (1967).

4. J. D. Fuelberth and M. L. Teply, A splitting ring of global dimension two, Proc. Amer. Math. Soc., 35 (1972), 317-324. 
5. E. R. Gentile, Singular submodule and injective hull, Indag. Math., 24 (1962), 426-433.

6. K. R. Goodearl, Singular torsion and the splitting properties, Amer. Math. Soc. Memoirs No. 124, (1972).

7. J. C. Robson, Idealizers and hereditary noetherian prime rings, J. Algebra, 22 (1972), 45-81.

8. J. C. Robson, Idealizer rings, in Ring Theory (R. Gordon, Ed.), New York (1972), Academic Press, 309-317.

Received February 11, 1972 and in revised form March 23, 1973.

UNIVERSity OF CHICAGO

Current Address: University of Utah, Salt Lake City, Utah 84112 


\section{PACIFIC JOURNAL OF MATHEMATICS}

\section{EDITORS}

RICHARD ARENS (Managing Editor)

University of California

Los Angeles, California 90024

\author{
R. A. Beaumont \\ University of Washington \\ Seattle, Washington 98105
}

J. Dugundu*

Department of Mathematics

University of Southern California

Los Angeles, California 90007

D. Gilbarg and J. Milgram

Stanford University

Stanford, California 94305

\section{ASSOCIATE EDITORS}
E. F. BECKENBACH
B. H. NeumanN
F. WOLF
K. YosHIDA

\section{SUPPORTING INSTITUTIONS}

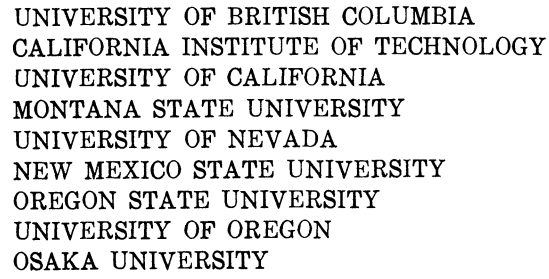

UNIVERSITY OF BRITISH COLUMBIA CALIFORNIA INSTITUTE OF TECHNOLOGY

UNIVERSITY OF CALIFORNIA

MONTANA STATE UNIVERSITY

UNIVERSITY OF NEVADA

NEW MEXICO STATE UNIVERSITY

OREGON STATE UNIVERSITY

UNIVERSITY OF OREGON

OSAKA UNIVERSITY

\author{
UNIVERSITY OF SOUTHERN CALIFORNIA \\ STANFORD UNIVERSITY \\ UNIVERSITY OF TOKYO \\ UNIVERSITY OF UTAH \\ WASHINGTON STATE UNIVERSITY \\ UNIVERSITY OF WASHINGTON \\ * * * \\ AMERICAN MATHEMATICAL SOCIETY \\ NAVAL WEAPONS CENTER
}

The Supporting Institutions listed above contribute to the cost of publication of this Journal, but they are not owners or publishers and have no responsibility for its content or policies.

Mathematical papers intended for publication in the Pacific Journal of Mathematics should be in typed form or offset-reproduced, (not dittoed), double spaced with large margins. Underline Greek letters in red, German in green, and script in blue. The first paragraph or two must be capable of being used separately as a synopsis of the entire paper. Items of the bibliography should not be cited there unless absolutely necessary, in which case they must be identified by author and Journal, rather than by item number. Manuscripts, in duplicate if possible, may be sent to any one of the four editors. Please classify according to the scheme of Math. Rev. Index to Vol. 39. All other communications to the editors should be addressed to the managing editor, or Elaine Barth, University of California, Los Angeles, California, 90024.

50 reprints are provided free for each article; additional copies may be obtained at cost in multiples of 50 .

The Pacific Journal of Mathematics is issued monthly as of January 1966. Regular subscription rate: $\$ 48.00$ a year (6 Vols., 12 issues). Special rate: $\$ 24.00$ a year to individual members of supporting institutions.

Subscriptions, orders for back numbers, and changes of address should be sent to Pacific Journal of Mathematics, 103 Highland Boulevard, Berkeley, California, 94708.

PUBLISHED BY PACIFIC JOURNAL OF MATHEMATICS, A NON-PROFIT CORPORATION

Printed at Kokusai Bunken Insatsusha (International Academic Printing Co., Ltd.), 270, 3-chome Totsuka-cho. Shinjuku-ku, Tokyo 160, Japan.

* C. R. DePrima California Institute of Technology, Pasadena, CA 91109, will replace J. Dugundji until August 1974.

Copyright (C) 1973 by

Pacific Journal of Mathematics

All Rights Reserved 


\section{Pacific Journal of Mathematics}

Vol. 48, No. $2 \quad$ April, 1973

Mir Maswood Ali, Content of the frustum of a simplex................

Mieczyslaw Altman, Contractors, approximate identities and factorization

in Banach algebras ................................ 323

Charles Francis Amelin, A numerical range for two linear operators ...... 335

John Robert Baxter and Rafael Van Severen Chacon, Nonlinear functionals

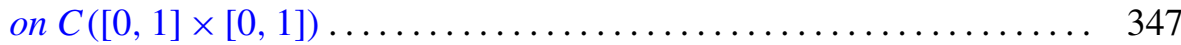

Stephen Dale Bronn, Cotorsion theories....................... 355

Peter A. Fowler, Capacity theory in Banach spaces............... 365

Jerome A. Goldstein, Groups of isometries on Orlicz spaces ........... 387

Kenneth R. Goodearl, Idealizers and nonsingular rings . ............ 395

Robert L. Griess, Jr., Automorphisms of extra special groups and

nonvanishing degree 2 cohomology ..................... 403

Paul M. Krajkiewicz, The Picard theorem for multianalytic functions . . . . 423

Peter A. McCoy, Value distribution of linear combinations of axisymmetric harmonic polynomials and their derivatives ...................

A. P. Morse and Donald Chesley Pfaff, Separative relations for

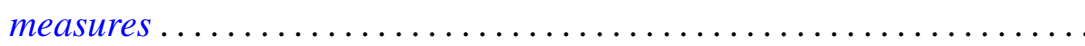

Albert David Polimeni, Groups in which $\operatorname{Aut}(G)$ is transitive on the

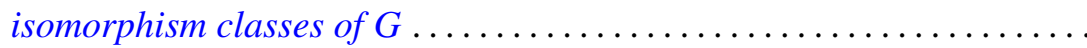

Aribindi Satyanarayan Rao, Matrix summability of a class of derived

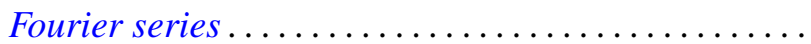

Thomas Jay Sanders, Shape groups and products

Ruth Silverman, Decomposition of plane convex sets. II. Sets associated

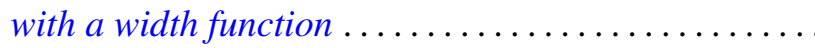

Richard Snay, Decompositions of $E^{3}$ into points and countably many flexible dendrites.............................

John Griggs Thompson, Nonsolvable finite groups all of whose local subgroups are solvable, IV ...

Robert E. Waterman, Invariant subspaces, similarity and isometric equivalence of certain commuting operators in $L_{p} \ldots$

James Chin-Sze Wong, An ergodic property of locally compact amenable

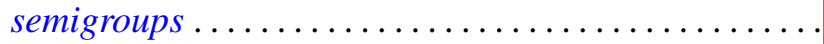

Julius Martin Zelmanowitz, Orders in simple Artinian rings are strongly equivalent to matrix rings ....................... 